\title{
Intestinal Stem Cells
}

\author{
Shahid Umar \\ Department of Internal Medicine, Division of Digestive Diseases, University of Oklahoma Health \\ Sciences Center, 975 NE 10th Street, SL Young BRC West 1268B, Oklahoma City, OK 73104, USA \\ Shahid Umar: Shahid-Umar@ouhsc.edu
}

\begin{abstract}
Self-renewal in the intestinal epithelia is fueled by a population of undifferentiated intestinal stem cells (ISCs) that give rise to daughter or progenitor cells, which can subsequently differentiate into the mature cell types required for normal gut function. The cellular signals that regulate self-renewal are poorly understood and the factors that mediate the transition from a stem cell to a progenitor cell in the gut are unknown. Recent studies have suggested that ISCs are located either at the crypt base interspersed between the Paneth cells (eg, Lgr-5+ve cells) or at or near position 4 within the intestinal crypt (eg, DCAMKL-1 or Bmi-1+ve cells). This raises the possibility that distinct stem cell regions exist in the crypts and that ISC's state of activation will determine how the self-renewal is regulated in the intestinal tract.
\end{abstract}

\section{Keywords}

Intestinal stem cell (ISC); Self-renewal; Transit amplifying (TA) cell; Enterocytes; Paneth cells; Enteroendocrine cells; Goblet cells; Intestinal crypts; Inflammation; Radiation

\section{Introduction}

The absorptive and protective functions of the gut are dependent on an intact and functional intestinal epithelium. Homeostasis of the normal adult intestinal epithelium is maintained by continuous and rapid replacement of differentiated cells by replication of undifferentiated epithelial or transit cells located within the crypts and subsequent differentiation of their progeny during migration away from the zone of replication. This renewal process involves rapid and continuous proliferation of epithelial cells in the crypt base with subsequent migration of these cells along the crypt-villus axis. The process of epithelial cell renewal within the intestine appears to be entirely dependent upon a limited number of long-lived multipotent intestinal stem or progenitor cells. An intestinal stem cell (ISC), similar to stem cells of the mouse hematopoietic system and the hair follicle, may be broadly defined by at least two properties: the ability to maintain itself throughout long periods of time (ie, self-renewal) and the potential to generate all differentiated cell types including enterocytes, goblet cells, enteroendocrine cells, and Paneth cells (ie, multipotency). When stem cells divide, they are believed to undergo an asymmetric cell division into a new stem cell plus a committed daughter cell. The rapidly cycling daughter cells, also called transit amplifying (TA) cells, then undergo a limited number of cell divisions before terminally differentiating into a tissue mass. The regulatory mechanisms that control stem cell proliferation at baseline and in response to injury are just beginning to be explored. This review highlights recent discoveries and implications

Correspondence to: Shahid Umar, Shahid-Umareouhsc . edu.

Disclosure No potential conflict of interest relevant to this article was reported. 
the ISC field may have on the regenerative processes in the digestive tract involved in health and disease.

\section{Structure and Function of Small Intestinal Crypts}

The intestinal epithelium is the most vigorously self-renewing tissue of adult mammals [1]. It is specified from endoderm formed during gastrulation and remains as a stratified cuboidal epithelium until mid-gestation in most vertebrates [2]. During middle to late gestation, the basic tissue architecture of the intestine is established through epithelial-mesenchymal interactions. Induced by signals from mesoderm-derived mesenchyme, the endoderm-derived epithelium evaginates to form villi and intervillus regions. The intervillus regions consist of undifferentiated and actively dividing cells that eventually invaginate into the mucosa to form the crypt of Lieberkühn in the first few days after birth and continue to develop during the next several weeks in the rodent [3].

The small intestinal architecture consists of continuous villi and crypts (Fig. 1a). The functional compartment of the epithelium contains differentiated cells no longer capable of dividing that have the features of mature epithelial cells. These differentiated epithelial cells populate the villi and can be categorized based on their function: enterocytes that function to absorb nutrients, goblet cells that secrete a protective mucus barrier and enteroendocrine cells that release gastrointestinal hormones (Fig. 1b). Paneth cells that secrete antibacterial peptides on the other hand, reside at the base of the proliferative compartment [4]. The proliferative compartment contains undifferentiated and rapidly cycling cells that populate the crypts of Lieberkühn. The epithelium in this region is responsible for providing the tremendous cell turnover and the protective niche for the epithelial stem cell. As cells begin to differentiate, they migrate toward the lumen and are eventually shed, either from the tip of the intestinal villi or from the surface of the colonic epithelium. Therefore, the crypt is mainly a proliferative compartment, is monoclonal, and is maintained by multipotent stem cells whereas the villus represents the differentiated compartment and is polyclonal as it receives cells from multiple crypts [5].

The well-compartmentalized structure of the small intestine is advantageous for analysis of developmental processes; however, study within this tissue has been limited by lack of a robust in vitro culture system for primary intestinal epithelial cells. Recently, studies with transgenic and knockout mice have shed light on molecular mechanisms underlying the fetal development of intestine as well as homeostatic epithelial regeneration in the adult. Through these studies, several signaling pathways such as the Wnt, bone morphogenic protein (BMP), phosphatidyl inositol $(3,4,5)$ kinase (PI3K), and Notch cascades have been revealed to play critical roles in regulating proliferation and controlling ISC self-renewal and differentiation in normal tissue.

\section{Stem/Progenitor Cells and Differentiated Cellular Lineages in the Crypt/Villi Axis of Small Intestinal Epithelium}

Stem cells are defined by their ability to self-renew and to give rise to mature cell types to maintain the integrity of the intestinal epithelium. Given the importance of stem cells to body tissues, it has long been postulated that stem cells should divide infrequently to prevent the acquisition of errors during DNA replication and to preserve their long-term proliferation potential. In humans, however, billions of cells are lost on a daily basis and therefore must be replenished for survival. Moreover, human tissues occasionally face traumatic injuries. The injured tissues have to be repaired quickly through rapid stem cell division and differentiation to maintain a proper balance. Thus, an extraordinary mechanism of regulation at the stem cell level apparently exists to allow stem cells to divide sparingly, to maintain their long-term potency, and rapidly, to maintain tissue homeostasis and repair injured tissues. Small intestinal 
epithelial lining is regenerated every 4 to 5 days. Either four to six [6] or one ISC [7] are present in small intestinal crypts, which cycle slowly (24-30 h) and give rise to rapidly cycling TA cells by asymmetric division [8]. The TA cells move up the crypt and undergo differentiation into either absorptive (enterocytes) or secretory lineages (mucous, enteroendocrine, Paneth cells), which then move upward into the villus [9] (Fig. 1). Paneth cells, however, migrate downward to the base of the crypts [4]. Enterocytes are the dominant lineage (90\% of total cells), goblet cells comprise $8 \%$ to $10 \%$ and enteroendocrine cells comprise about $1 \%$ of the epithelium. Stem cells in animal tissues are often located and controlled by special tissue microenvironments known as niches. A stem cell niche can be defined as "a specific location in a tissue where stem cells can reside for an indefinite period of time and produce progeny cells while self-renewing." This niche is made up of and influenced by nearby proliferating and differentiating epithelial cells as well as by surrounding mesenchymal cells. Although separated from the epithelial cells by the basement membrane, these mesenchymal cells (ie, blood vessels, intraepithelial lymphocytes, and fibroblasts/myofibroblasts) together with the extracellular matrix, promote the epithelial-mesenchymal crosstalk required to maintain the stem cell niche. Epithelial-mesenchymal interactions enable specification of epithelial stem cells during development [10], but the requirement and nature of these communications in adult stem cells remain to be characterized. Excessive stem cell production can result in cancer; thus, maintaining a balance of stem cell quiescence and activity is a hallmark of a functional niche [11]. We have just begun to understand which niche signals regulate self-renewal and maintain the balance between self-renewal and differentiation of ISCs. An increasing number of signaling pathways, including Wnt, BMP, Hedgehog, and Notch, may play important roles in this regard, as discussed in the following section.

\section{Signaling Pathways and ISC}

Wnt proteins form a family of highly conserved secreted signaling molecules that are capable of signaling both in an autocrine and paracrine fashion. Wnt induces its biologic effects by binding to either the seven-span transmembrane protein Frizzled (Fz) or the single-span lowdensity lipoprotein receptor-related protein (LRP). Wnt-mediated signaling results in increased stability of $\beta$-catenin in the cytoplasm, and its interaction with the T-cell factor 4 (Tcf-4) transcription factor within the nucleus. Under normal conditions, $\beta$-catenin forms a complex of proteins containing adenomatosis polyposis coli (APC), axin, and glycogen synthase kinase- $3 \beta$ (GSK-3 $\beta$ ) and its levels are tightly controlled through ubiquitin-mediated proteasomal degradation. Wnt factors inhibit the $\beta$-catenin-APC complex. Hence, $\beta$-catenin is stabilized and interacts with nuclear Tcf-4 to drive the transcription of specific target genes. Initial studies demonstrated that active Wnt signaling was found in intervillus regions and was thought to inhibit differentiation and stimulate proliferation, as indicated by experiments in which inhibition of Wnt signaling by Tcf- 4 disruption halted the proliferation of endoderm and depleted endodermal stem/progenitor cells [12]. These experiments also showed that proliferative cells in the intervillus region were replaced by mature enterocytes usually seen in the villus [12]. However, recent findings indicate that Wnt signaling within the developing intestine may be more complex. In fact, Wnt activity was found exclusively within the villus epithelium from embryonic day 16 (E16) to postnatal day 2 (P2) and was not seen within the intervillus region until P2. However, by E14, villus and intervillus regions displayed unique genetic signatures and by E17.5, proliferative cells were restricted to intervillus regions. In addition, although nuclear $\beta$-catenin and c-MYC expression corresponded to the villus cells with Wnt activity, other known Wnt target genes and pathway components such as CD44, CyclinD1, and Tcf-4 were present within the intervillus region [13]. These results suggest that Wnt signaling and proliferation may be uncoupled during early development and that perhaps Wnt-independent mechanisms drive early epithelial proliferation. Furthermore, Wnt signaling may play an important role in initial villus formation. Wnt signaling components are expressed by crypt epithelial cells and surrounding mesenchymal cells, which play an extremely 
important role in normal homeostasis $[11,14,15]$ (Fig. 1). Wnt inhibitors, such as Dkk3, may be expressed in a graded manner along the crypt-villus axis, providing the balance between positive and negative regulators of this pathway [16].

Indian hedgehog (Ihh) is also expressed in the intervillus region, whereas its receptor, Patched, is expressed in the adjacent mesoderm [17]. Because Hedgehog inhibition compromises villus formation, this signal is thought to work as a morphogen within the intestine as in other tissues [18]. It remains unclear whether Ihh signaling is dependent on Wnt activation or is completely independent. More recently, Ihh was shown to regulate ISC self-renewal and differentiation [19•]. Intestinal epithelial Ihh signals to the mesenchymal compartment to regulate formation and proliferation of mesenchymal cells, which in turn affects the epithelial proliferation and differentiation. These findings provide a basis for analyses of the role of the muscularis mucosae in ISC regulation. Indeed, expression of BMPs mainly in mesoderm-derived mesenchymal cells is regulated by Hedgehog signaling, and BMP signaling also plays a role in regulating morphogenesis during intestinal development [20]. Inhibition of BMP signaling by over-expression of its inhibitor, Noggin, or conditional inactivation of its receptor,

BMPR1A, causes ectopic crypt formation, suggesting a role for BMP signaling in restricting crypt numbers [21].

A high level of Notch is expressed in the intestinal stem cells [11]. Four isoforms of the Notch receptor have been identified in mammals (Notch1-4) [22]. Notch activation is achieved through direct interaction with specific transmembrane ligands of the DSL (Delta/Serrate/ Caenorhabditis elegans Lag-2) family, such as Delta-like (DLL) 1, 3, and 4 and Jagged 1-2 in mammals [23]. Notch-DSL ligand binding initiates proteolytic cleavage at both the extracellular and intracellular regions of the Notch receptor, leading to the release of Notch intracellular domain (NICD) [24]. Nuclear localization signals within the NICD direct its translocation, where it binds to and displaces a CSL transcriptional corepressor complex [25, 26]. Basic helix-loop-helix transcription factors of the Hairy/E(spl) (HES) and "HES-related genes in vertebrates" class are the best-characterized Notch targets [27]. Importantly, Notch transcriptional output depends greatly upon specific cell and tissue types because various events such as pathway crosstalk can affect the transcriptional regulation of tissue-specific differentiation, development, and cell-cycle regulation [28].

The Notch signal controls daughter cells differentiating into absorptive rather than secretory cells. Like Wnt signaling, the Notch pathway also plays a role in stem cell maintenance [28]. Components of the Notch pathway are chiefly expressed in the epithelium at the crypt base, in the stem cell region. Increasing Notch signaling through forced expression of NICD in newborn mouse results in increased cell proliferation in the stem cell compartment and also shows a severe reduction of all three secretory cell types [28]. Conversely, inhibition of the Notch signaling in the intestinal epithelium, either by deletion of the Hes1 gene, the CSL gene, the Notch1 and Notch2 genes, or through pharmacologic $\gamma$-secretase inhibitors, results in an excessive number of secretory cells $[1,29]$. Although Notch signaling plays an important role in stem cell proliferation, the experimental results suggest that Notch signaling functions in the TA compartment controlling absorptive rather than secretory cell fate decisions in the intestinal epithelium.

In summary, among the four major signals characterized so far (Wnt, Notch, Hh, and BMP), Wnt signaling mainly functions in the crypt base to maintain stem cell proliferation and selfrenewal; Notch signaling mainly functions in the TA compartment to control daughter cell fate determination; and the Hh-BMP signaling from the crypt or intervillus pocket delivers a longrange signal to inhibit the formation of crypts and promote the formation of villi. 


\section{Identification of Stem Cells/Stem Cell Markers in Small Intestinal Crypts}

The location, number, and behavior of ISCs within the base of the crypt have been characterized by numerous investigators using murine models [4], following chemoradiation [30], somatic mutation [31], chimeric breeding [32], and transgenic overexpression or ablation of specific regulatory genes $[12,20,21,33]$. Interpretation of these data with respect to the precise position and behavior of ISC within the intestinal crypt continues to be debated [34,35]. In the mouse small intestine, two types of stem cells have been identified [35]. One type is located below the +4 position in the "stem cell zone" and the other type is located at the +4 position from the crypt bottom. Cheng and Leblond [4] identified small cycling epithelial cells interspersed between the Paneth cells, or the so-called crypt base columnar (CBC) cells, using morphologic methods in mammalian intestine. Later, Bjerknes and Cheng [36] provided additional information on these specialized cells using elegant clonal marking techniques. These investigators postulated that the CBCs located within the stem cell zone of the crypt base might represent the actual ISC and that all the differentiated intestinal epithelial cell types develop from these CBCs [36]. An alternative hypothesis also suggested that the ISCs were actually located elsewhere at a position that averaged +4 from the bottom of the crypts, with the lowest three positions generally relegated to the terminally differentiated Paneth cells. Evidence supporting this hypothesis of the +4 stem cell model was provided by Potten [30]. These investigators, using the DNA-labeling reagents, bromodeoxyuridine or $\left({ }^{3} \mathrm{H}\right)$-thymidine, on radiation-sensitive, label-retaining cells (LRC) showed that the LRCs were located specifically at the +4 position in the intestinal crypt region, precisely at the origin of the migratory epithelial cell column.

One major obstacle in ISC biology has been the lack of definitive markers that identify ISCs. Reliable markers would allow for definitive identification of the stem cell population and would facilitate the ability to isolate and manipulate these cells in vitro. Numerous proteins are expressed in the crypt compartment but not in the villus, such as EphB2 [37], CD44 [38], and Hes1 [39]. Immunohistochemistry and in situ hybridization studies suggested that RNA binding protein Musashi-1 (Msi-1), involved in Notch signaling, is also expressed in the supraPaneth cells as well as in CBC cells (Table 1) [39,40]. Members of BMP and Wnt signaling pathways ( $p$-PTEN and $p$-AKT) localize to ISCs $[20,41]$. In recent years, fluorescenceactivated cell sorting has been used to identify side population (SP) cells in murine small intestine as a source of putative ISCs [42]. These SP cells were distinct from the hematopoietic stem cells and their progeny, based on the absence of surface markers for CD45, c-kit, and CD34. RNA prepared from CD45-negative intestinal SP cells were found to be enriched with Msi-1 [42]. The molecular features of the potential stem cell populations in SP faction were recently characterized [43]. Protein markers such as EphB2 [37], CD44 [38], Fgfr3 [44] and Sox9 [45] have a crypt-base expression pattern, but are not specific to ISCs. Analyses of various Wnt pathway components and their target genes have identified Sfrp5 [46] and Ascl2 [46] as possible ISC markers. Besides these, it was recently shown that telomerase reverse transcriptase (mTert), as identified in GFP transgenic mice, stains a few cells at cell position 4 [47]. None of these genes, however, encode for proteins that are amenable to cell-sorting procedures. Laser-capture microdissection method found numerous transcripts involved in cmyc signaling to be significantly expressed in putative ISC cells [48]. Immunohistochemistry studies showed that doublecortin and $\mathrm{Ca}^{2+} /$ calmodulin-dependent kinase-like-1 (DCAMKL-1) and Mapk14 are expressed within the stem cell zones [49].

Long-term DNA-label retention tentatively located stem cells at position +4 directly above Paneth cells [50]. Leucine-rich-repeat-containing G-protein-coupled receptor-5 (LGR5) expression was demonstrated in crypts of small intestines, but not in the villi by in situ hybridization [51••]. LGR5 gene marked the slender cycling crypt base columnar (CBC) cells (Fig. 1), interspersed between Paneth cells [51••]. LGR5 encodes an orphan G-protein-coupled 
receptor, characterized by a large leucine-rich extracellular domain [51••], and is expressed in colorectal, ovarian, and hepatocellular carcinomas [51••]. The CBC cells were positive for proliferation marker Ki67 and occasionally expressed M-phase marker phospho-histone H3 [51••]. Adult mice irradiated with 1 to $10 \mathrm{~Gy}$ were analyzed for apoptosis after $6 \mathrm{~h}$. Apoptosis was determined in $+4 \mathrm{CBC}$ cells and TA cells (located at position 5-15) [50]. Maximal apoptosis at +4 position was reached at $1 \mathrm{~Gy}$, whereas $10 \mathrm{~Gy}$ was needed to measure apoptosis in CBC and TA cells, suggesting that LGR5 may be a marker for stem cells in small and large intestines [51••]. Moreover, using genetic approaches, it was demonstrated that LGR5-positive CBCs are multipotent for all mature intestinal epithelial cell types, cycle every $24 \mathrm{~h}$, and persist for 60 days [51••]. Thus, even though questions remain regarding the functional role of LGR5 in the intestinal epithelia [52], loss of LGR5 may affect both crypt regeneration and neoplastic transformation.

In 2009, lineage-tracing studies of adult prominin-1 (also called CD133; a pentaspan transmembrane glycoprotein that localizes to membrane protrusions) showed that some prominin-1-positive cells are located at the base of crypts in the small intestine, co-express LGR5, and can generate the entire intestinal epithelium, and therefore seem to be small intestinal stem cells as well [53]. Moreover, olfacto medin 4 (OLFM4), which was identified in a gene expression profile for LGR5-positive cells, was shown to be highly expressed in CBCs in the human small intestine and colon, and may therefore be a marker for human intestinal and colon stem cells [54]. Sangiorgi and Capecchi [55••] characterized the progeny of crypt Bmi1-positive cells (Fig. 1c;Table 1) and argued in support of the +4 LRCs as a population of stem cells within the small intestine. Bmil encodes a chromatin remodeling protein of the polycomb group that has essential roles in self-renewal of hematopoietic and neural stem cells. Bmil seems to consistently mark long-lived cell clones (>12 months) populated by all intestinal lineages and serves as a specific marker of a cell population located at the +4 position of the crypt. Furthermore, ablation of Bmi1-positive cells by targeted expression of the diphtheria toxin depletes the epithelium of the genetically marked crypts (known as whole crypt units) [55••]. Thus, expression of Bmi1 also identifies ISC candidates. In addition to LGR5 and prominin-1, other potential stem cell markers have been identified for which lineage tracing is not yet complete.

It was reported recently that DCAMKL-1, a microtubule-associated kinase expressed in postmitotic neurons, is a novel putative ISC marker (Fig. 1c; Table 1) [56••]. DCAMKL-1 was identified as a Gene Ontogeny-enriched transcript expressed in comparison with gastric epithelial progenitor and whole stomach libraries [49] and more recently in gastric stem cells [57]. Immunoreactive DCAMKL-1 cells were found at or near position +4 , at a frequency of one cell per five crypts [56••]. DCAMKL-1-positive CBC cells were also observed, but at much lower frequency [56••]. More recently it was shown that the novel putative ISC marker DCAMKL-1 is predominantly expressed in quiescent cells in the lower two thirds of the small intestinal crypt epithelium and in occasional CBC cells [58•]. In contrast, novel putative stem cell marker LGR5 is observed in rapidly cycling CBCs and in occasional crypt epithelial cells [58•]. Functionally quiescent DCAMKL-1-positive crypt epithelial cells retained BrdU label in a modified label-retention assay [58•]. Importantly, it was demonstrated that DCAMKL-1 is a cell surface expression protein, and a specific antibody against the extracellular domain of DCAMKL-1 was used to isolate DCAMKL-1- positive cells from adult mouse small intestine by fluorescence-activated cell sorting [58•]. DCAMKL-1-positive cells were found to selfrenew and form spheroids in suspension culture [58•]. These spheroids formed glandular epithelial structures in the flanks of athymic nude mice, which expressed multiple markers of gut epithelial lineage [58•]. Thus, DCAMKL-1 is a marker of quiescent ISCs and can be distinguished from the cycling stem/progenitor cells that are LGR5 positive. DCAMKL-1 did not co-localize with other key markers such as chromogranin $\mathrm{A}, p \mathrm{PTEN}, p \mathrm{AKT}$, somatostatin, or secretin [58•]. LGR5 identifies proliferative CBC and TA cells in the gut as evidenced by 
co-labeling with proliferating cell nuclear antigen. Thus, the original hypothesis of a +4 ISC [50] is likely accurate, and DCAMKL-1-positive cells likely represent the elusive quiescent ISCs.

\section{Effect of Injury on Intestinal Stem Cells}

Restoration of normal epithelial architecture and function after intestinal injury induced by a variety of noxious agents (chemical, infectious, radiation, and inflammatory) is a multistep process that involves tight regulatory control of epithelial stem cell dynamics [59]. First, migration of adjacent epithelial cells over the wound reestablishes continuity of the epithelium. Stem cells divide to increase their numbers and to give rise to the more rapidly proliferating TA cells. The transit cell population then expands rapidly to form a regenerative crypt. A single surviving clonogenic crypt stem cell is sufficient to facilitate crypt regeneration. If the injury has completely destroyed some crypts, the surviving crypt stem cells can divide to increase their numbers, and subsequently restore sufficient numbers of crypts by crypt fission, to maintain epithelial homeostasis [59]. Whether this process involves recruitment of a quiescent stem cell population from within the crypt-villus axis or requires replenishment from the bone marrow remains to be determined. Moreover, because the epithelial stem cells within a crypt segregate to daughter crypts when crypt fission occurs, an expansion of the stem cell number must accompany this reparative response. However, the factors that coordinate this complex process in diseases such as human inflammatory bowel disease have not been fully elucidated, and much remains unknown about the biologic consequences of chronic intestinal inflammation on the fate or proliferation of the stem cell population.

\section{Inflammation}

Inflammatory bowel disease is characterized by cycles of mucosal injury and ulceration, followed by epithelial regeneration and restoration of normal epithelial architecture and function. In several mouse models of inflammatory bowel disease, alterations of epithelial function have been implicated in the pathogenesis of chronic inflammation [60]. In general, intestinal inflammation is associated with significant upregulation of pro-inflammatory cytokines, such as interleukin (IL)-1, IL-6, IL-8, IL-12 and tumor necrosis factor (TNF)- $\alpha$, along with other mediators and growth factors, such as cyclo-oxygenase (COX)-2, basic fibroblast growth factor (bFGF), hepatocyte growth factor, and vascular endothelial growth factor [60]. Expression of these inflammatory mediators is regulated by activation of nuclear factor- $\kappa \mathrm{B}$ (NF- $\mathrm{\kappa B})$. NF- $\mathrm{\kappa B}$ is a key coordinator of innate immunity and inflammation and has emerged as an important endogenous tumor promoter [61,62]. Crohn's ileitis is also associated with a reduced expression of Tcf-4, which regulates Paneth cell differentiation, leading to defects in innate immunity [63].

Expansion of the proliferative zone, increased crypt branching, and changes in the normal patterns of cellular differentiation have been documented in human inflammatory bowel disease [60], suggesting that the pathways regulating the relationship between cellular replication and epithelial differentiation have been altered in these diseases. Although it is clear that the intestinal epithelium responds to inflammation and mucosal injury by initiating a regenerative response, the specific effects inflammation may have on the in vivo turnover of epithelial stem cells or progenitor cells, or the way in which the inflammatory milieu may perturb normal epithelial differentiation and/or function remain obscure. Similarly, a number of cytokines, growth factors and other mediators including TNF- $\alpha$, transforming growth factor (TGF) 33 , trefoil factor-3 (TFF3), IL-1, IL-11, COX1/2, bFGF, FGF-7, and FGF-10 associated with intestinal inflammation or injury can induce increase in crypt epithelial stem cell survival when exogenously administered before radiation $[64,65]$. However, more work is needed to understand how enhanced expression of one or more of these peptides, as a consequence of ileal inflammation, directly affects epithelial stem cells or differentiation of progenitor cells. 


\section{Radiation}

The radiosensitivity of the small intestine is primarily a function of rapidly dividing progenitor cells derived from epithelial stem cells near the base of intestinal crypts. Although crypt epithelial cells are extremely sensitive to radiation-induced apoptosis, there is little to no apoptosis in the villi. Doses as low as 0.01 to $1.0 \mathrm{~Gy}$ induce apoptosis within the lower crypt. Peak apoptosis is observed between 3 and $6 \mathrm{~h}$ post-irradiation [65-67]. However, at higher doses (>8 Gy), a second apoptotic wave occurs $24 \mathrm{~h}$ post-irradiation. After severe irradiation, epithelial cell loss at the villus tips creates an imbalance between epithelial production and turnover, resulting in villi shortening and disruption of the mucosal barrier if all or most of the crypt stem cells die. However, if a single stem cell survives in the crypt region, regenerative crypts will appear within 3 to 4 days, and crypt-villi architecture will be restored within 6 to 8 days. The balance between the rate and extent of crypt-villi regeneration, which depends on the stem cell and the pathologic consequences of denuding the epithelial covering from the small intestine, is a major determinant of survival in the early period after acute radiation exposure.

\section{Conclusions}

The possible relationship between the quiescent and the actively cycling nature of the ISCs needs to be further explored. Moreover, it is critical to understand the genetic elements that determine stem cell fate and the basis by which regeneration occurs in order to better understand stem cell plasticity and the contribution made by the stem cell compartment to malignant disease. It is hoped that future studies in this area will provide a better platform to develop therapies to regenerate damaged intestinal epithelia as seen after radiation injuries or inflammatory bowel disease (eg, Crohn's disease).

\section{Acknowledgments}

This work was partially supported by National Institutes of Health Grant R01 CA131413 (to SU) from the NCI.

\section{References}

Papers of particular interest, published recently, have been highlighted as:

- Of importance

•• Of major importance

1. Crosnier C, Stamataki D, Lewis J. Organizing cell renewal in the intestine: stem cells, signals and combinatorial control. Nat Rev Genet 2006;7:349-359. [PubMed: 16619050]

2. de Santa Barbara P, van den Brink GR, Roberts DJ. Development and differentiation of the intestinal epithelium. Cell Mol Life Sci 2003;60:1322-1332. [PubMed: 12943221]

3. Schmidt GH, Winton DJ, Ponder BA. Development of the pattern of cell renewal in the crypt-villus unit of chimeric mouse small intestine. Development 1988;103:785-790. [PubMed: 3248525]

4. Cheng H, Leblond CP. Origin, differentiation and renewal of the four main epithelial cell types in the mouse small intestine. V. Unitarian Theory of the origin of the four epithelial cell types. Am J Anat 1974;141:537-561. [PubMed: 4440635]

5. Wright NA. Epithelial stem cell repertoire in the gut: clues to the origin of cell lineages, proliferative units and cancer. Int J Exp Pathol 2000;81:117-143. [PubMed: 10762441]

6. Potten CS, Loeffler M. Stem cells: attributes, cycles, spirals, pitfalls and uncertainties. Lessons for and from the crypt. Development 1990;110:1001-1020. [PubMed: 2100251]

7. Gordon JI, Schmidt GH, Roth KA. Studies of intestinal stem cells using normal, chimeric, and transgenic mice. FASEB J 1992;6:3039-3050. [PubMed: 1521737] 
8. Potten CS. Stem cells in gastrointestinal epithelium: numbers, characteristics and death. Philos Trans R Soc Lond B Biol Sci 1998;353:821-830. [PubMed: 9684279]

9. Bjerknes M, Cheng H. The stem-cell zone of the small intestinal epithelium. I. Evidence from Paneth cells in the adult mouse. Am J Anat 1981;160:51-63. [PubMed: 7211716]

10. Blanpain C, Horsley V, Fuchs E. Epithelial stem cells: turning over new leaves. Cell 2007;128:445458. [PubMed: 17289566]

11. Moore KA, Lemischka IR. Stem cells and their niches. Science 2006;311:1880-1885. [PubMed: 16574858]

12. Korinek V, Barker N, Moerer P, et al. Depletion of epithelial stem-cell compartments in the small intestine of mice lacking Tcf-4. Nat Genet 1998;19:379-383. [PubMed: 9697701]

13. Kim BM, Mao J, Taketo MM, et al. Phases of canonical Wnt signaling during the development of mouse intestinal epithelium. Gastroenterology 2007;133:529-538. [PubMed: 17681174]

14. van de Wetering M, Sancho E, Verweij C, et al. The $\beta$-catenin/TCF-4 complex imposes a crypt progenitor phenotype on colorectal cancer cells. Cell 2002;111:241-250. [PubMed: 12408868]

15. Gregorieff A, Pinto D, Begthel H, et al. Expression pattern of Wnt signaling components in the adult intestine. Gastroenterology 2005;129:626-638. [PubMed: 16083717]

16. Byun T, Karimi M, Marsh JL, et al. Expression of secreted Wnt antagonists in gastrointestinal tissues: potential role in stem cell homeostasis. J Clin Pathol 2005;58:515-519. [PubMed: 15858124]

17. Madison BB, Braunstein K, Kuizon E, et al. Epithelial hedgehog signals pattern the intestinal cryptvillus axis. Development 2005;132:279-289. [PubMed: 15590741]

18. Bitgood MJ, McMahon AP. Hedgehog and Bmp genes are co-expressed at many diverse sites of cellcell interaction in the mouse embryo. Dev Biol 1995;172:126-138. [PubMed: 7589793]

19•. Zacharias WJ, Li X, Madison BB, et al. Hedgehog is an antiinflammatory epithelial signal for the intestinal lamina propria. Gastroenterology 2010;138:2368-2377. [PubMed: 20206176] This article investigates the effects of chronic Hedgehog $(\mathrm{Hh})$ inhibition in vivo by profiling molecular pathways acutely modulated by Hh signaling in the intestinal mesenchyme.

20. He XC, Zhang J, Tong WG, et al. BMP signaling inhibits intestinal stem cell self-renewal through suppression of Wnt- $\beta$-catenin signaling. Nat Genet 2004;36:1117-1121. [PubMed: 15378062]

21. Haramis AP, Begthel H, van den Born M, et al. De novo crypt formation and juvenile polyposis on BMP inhibition in mouse intestine. Science 2004;303:1684-1686. [PubMed: 15017003]

22. Louvi A, Artavanis-Tsakonas S. Notch signaling in vertebrate neural development. Nat Rev Neurosci 2006;7:93-102. [PubMed: 16429119]

23. Chiba S. Notch signaling in stem cell systems. Stem Cells 2006;24:2437-2447. [PubMed: 16888285]

24. Fortini ME. Gamma-secretase-mediated proteolysis in cell-surface-receptor signaling. Nat Rev Mol Cell Biol 2002;3:673-684. [PubMed: 12209127]

25. Kao HY, Ordentlich P, Koyano-Nakagawa N, et al. A histone deacetylase corepressor complex regulates the Notch signal transduction pathway. Genes Dev 1998;12:2269-2277. [PubMed: 9694793]

26. Lai EC. Keeping a good pathway down: transcriptional repression of Notch pathway target genes by CSL proteins. EMBO Rep 2002;3:840-845. [PubMed: 12223465]

27. Iso T, Kedes L, Hamamori Y. HES and HERP families: multiple effectors of the Notch signaling pathway. J Cell Physiol 2003;194:237-255. [PubMed: 12548545]

28. Fre S, Huyghe M, Mourikis P, et al. Notch signals control the fate of immature progenitor cells in the intestine. Nature 2005;435:964-968. [PubMed: 15959516]

29. van der Flier LG, Clevers H. Stem cells, self-renewal, and differentiation in the intestinal epithelium. Annu Rev Physiol 2008;71:241-260. [PubMed: 18808327]

30. Potten CS. Extreme sensitivity of some intestinal crypt cells to X and gamma irradiation. Nature 1977;269:518-521. [PubMed: 909602]

31. Potten CS, Owen G, Booth D. Intestinal stem cells protect their genome by selective segregation of template DNA strands. J Cell Sci 2002;115:2381-2388. [PubMed: 12006622]

32. Winton DJ, Blount MA, Ponder BAJ. A clonal marker induced by mutation in mouse intestinal epithelium. Nature 1988;333:463-466. [PubMed: 3163778] 
33. He XC, Yin T, Grindley JC, et al. PTEN-deficient intestinal stem cells initiate intestinal polyposis. Nat Genet 2007;39:189-198. [PubMed: 17237784]

34. Barker N, van de Wetering M, Clevers H. The intestinal stem cell. Genes Dev 2008;22:1856-1864. [PubMed: 18628392]

35. Scoville DH, Sato T, He XC, et al. Current view: intestinal stem cells and signaling. Gastroenterology 2008;134:849-864. [PubMed: 18325394]

36. Bjerknes M, Cheng H. Clonal analysis of mouse intestinal epithelial progenitors. Gastroenterology 1999;116:7-14. [PubMed: 9869596]

37. Batlle E, Henderson JT, Beghtel H, et al. Beta-catenin and TCF mediate cell positioning in the intestinal epithelium by controlling the expression of EphB/ephrinB. Cell 2002;111:251-263. [PubMed: 12408869]

38. Wielenga VJ, Smits R, Korinek V, et al. Expression of CD44 in Apc and Tcf mutant mice implies regulation by the WNT pathway. Am J Pathol 1999;154:515-523. [PubMed: 10027409]

39. Kayahara T, Sawada M, Takaishi S, et al. Candidate markers for stem and early progenitor cells, Musashi-1 and Hes 1, are expressed in crypt base columnar cells of mouse small intestine. FEBS Lett 2003;535:131-135. [PubMed: 12560091]

40. Potten CS, Booth C, Tudor GL, et al. Identification of a putative intestinal stem cell and early lineage marker musashi-1. Differentiation 2003;71:28-41. [PubMed: 12558601]

41. Tian Q, Feetham MC, Tao WA, et al. Proteomic analysis identifies that 14-3-3zeta interacts with beta-catenin and facilitates its activation by Akt. Proc Natl Acad Sci U S A 2004;101:15370-15375. [PubMed: 15492215]

42. Dekaney CM, Rodriguez JM, Graul MC, et al. Isolation and characterization of a putative intestinal stem cell fraction from mouse jejunum. Gastroenterology 2005;129:1567-1580. [PubMed: 16285956]

43. Gulati AS, Ochsner SA, Henning SJ. Molecular properties on side population-sorted cells from mouse small intestine. Am J Physiol Gastrointest Liver Physiol 2008;294:G286-G294. [PubMed: 18006601]

44. Vidrich A, Buzan JM, Ilo C, et al. Fibroblast growth factor receptor-3 is expressed in undifferentiated intestinal epithelial cells during murine crypt morphogenesis. Dev Dyn 2004;230:114-123. [PubMed: 15108315]

45. Blache P, van de Wetering M, Duluc I, et al. SOX9 is an intestine crypt transcription factor, is regulated by the Wnt pathway, and represses the CDX2 and MUC2 genes. J Cell Biol 2004;166:37-47. [PubMed: 15240568]

46. Van der Flier LG, Sabates-Bellver J, Oving I, et al. The intestinal Wnt/TCF signature. Gastroenterology 2007;132:628-632. [PubMed: 17320548]

47. Breault DT, Min IM, Carlone DL, et al. Generation of mTert-GFP mice as a model to identify and study tissue progenitor cells. Proc Natl Acad Sci U S A 2008;105:10420-10425. [PubMed: 18650388]

48. Stappenbeck TS, Mills JC, Gordon JI. Molecular features of adult mouse small intestinal epithelial progenitors. Proc Natl Acad Sci U S A 2003;100:1004-1009. [PubMed: 12552106]

49. Giannakis M, Stappenbeck TS, Mills JC, et al. Molecular properties of adult mouse gastric and intestinal epithelial progenitors in their niches. J Biol Chem 2006;281:11292-11300. [PubMed: 16464855]

50. Potten CS, Booth C, Pritchard DM. The intestinal epithelial stem cell: the mucosal governor. Int J Exp Pathol 1997;78:219-243. [PubMed: 9505935]

$51 \bullet$. Barker N, van Es JH, Kuipers J, et al. Identification of stem cells in small intestine and colon by marker gene LGR5. Nature 2007;449:1003-1008. [PubMed: 17934449] Using knock-in alleles, this elegant study identifies stem cells in small intestine and colon by marker gene Lgr5.

52. Potten CS, Gandara R, Mahida YR, et al. The stem cells of small intestinal crypts: where are they? Cell Prolif 2009;42:731-750. [PubMed: 19788585]

53. Zhu L, Gibson P, Currle DS, Tong Y, et al. Prominin 1 marks intestinal stem cells that are susceptible to neoplastic transformation. Nature 2009;457:603-607. [PubMed: 19092805] 
54. van der Flier LG, Haegebarth A, Stange DE, et al. OLFM4 is a robust marker for stem cells in human intestine and marks a subset of colorectal cancer cells. Gastroenterology 2009;137:15-17. [PubMed: 19450592]

55••. Sangiorgi E, Capecchi MR. Bmi1 is expressed in vivo in intestinal stem cells. Nat Genet 2008;40:915-920. [PubMed: 18536716] Using a mouse expressing a tamoxifen-inducible Cre from the Bmil locus, this study identifies Bmil as an ISC marker in vivo.

56••. May R, Riehl TE, Hunt C, et al. Identification of a novel putative gastrointestinal stem cell and adenoma stem cell marker, doublecortin and CaM kinase-like-1, following radiation injury and in adenomatous polyposis coli/multiple intestinal neoplasia mice. Stem Cells 2008;26:630-637. [PubMed: 18055444] Using the radiation injury and APC/Min mouse models, this study identifies DCAMKL-1 as an ISC marker in vivo.

57. Giannakis M, Chen SL, Karam SM, et al. Helicobacter pylori evolution during progression from chronic atrophic gastritis to gastric cancer and its impact on gastric stem cells. Proc Natl Acad Sci U S A 2008;105:4358-4363. [PubMed: 18332421]

58•. May R, Sureban SM, Hoang N, et al. Doublecortin and CaM kinase-like-1 and leucine-rich-repeatcontaining G-protein-coupled receptor mark quiescent and cycling intestinal stem cells, respectively. Stem Cells 2009;27:2571-2579. [PubMed: 19676123] Using the modified labelretention assay, this study compares the quiescent versus actively cycling nature of the intestinal stem markers DCAMKL-1 and Lgr5, respectively.

59. Potten CS. A comprehensive study of the radiobiological response of the murine (BDF1) small intestine. Int J Rad Biol 1990;58:925-973. [PubMed: 1978853]

60. Pizarro TT, Arseneau KO, Cominelli F. Lessons from genetically engineered animal models XI Novel mouse models to study pathogenic mechanisms of Crohn's disease. Am J Physiol 2000;278:G665G669.

61. Jobin C, Sartor RB. The I kappa B/NF-kappa B system: a key determinant of mucosal inflammation and protection. Am J Physiol 2000;278:C451-C462.

62. Karin M. Nuclear factor- $\kappa B$ in cancer development and progression. Nature 2006;441:431-436. [PubMed: 16724054]

63. Wehkamp J, Wang G, Kubler I, et al. The Paneth cell alpha defensin deficiency of ileal Crohn's disease is linked to Wnt/Tcf-4. J Immunol 2007;179:3109-3118. [PubMed: 17709525]

64. Riehl T, Cohn S, Tessner T, et al. Lipopolysaccharide is radioprotective in the mouse intestine through a prostaglandin-mediated mechanism. Gastroenterology 2000;118:1106-1116. [PubMed: 10833485]

65. Houchen CW, George RJ, Sturmoski MA, et al. FGF-2 enhances intestinal stem cell survival and its expression is induced after radiation injury. Am J Physiol 1999;276:G249-G258. [PubMed: 9887002]

66. Wu S, Miyamoto T. Radioprotection of the intestinal crypts of mice by recombinant human interlukin-1. Radiat Res 1990;123:112-115. [PubMed: 2371377]

67. Somosy Z, Horvath G, Telbisz A, et al. Morphological aspects of ionizing radiation response of small intestine. Micron 2002;33:167-178. [PubMed: 11567886]

Curr Gastroenterol Rep. Author manuscript; available in PMC 2011 October 1. 


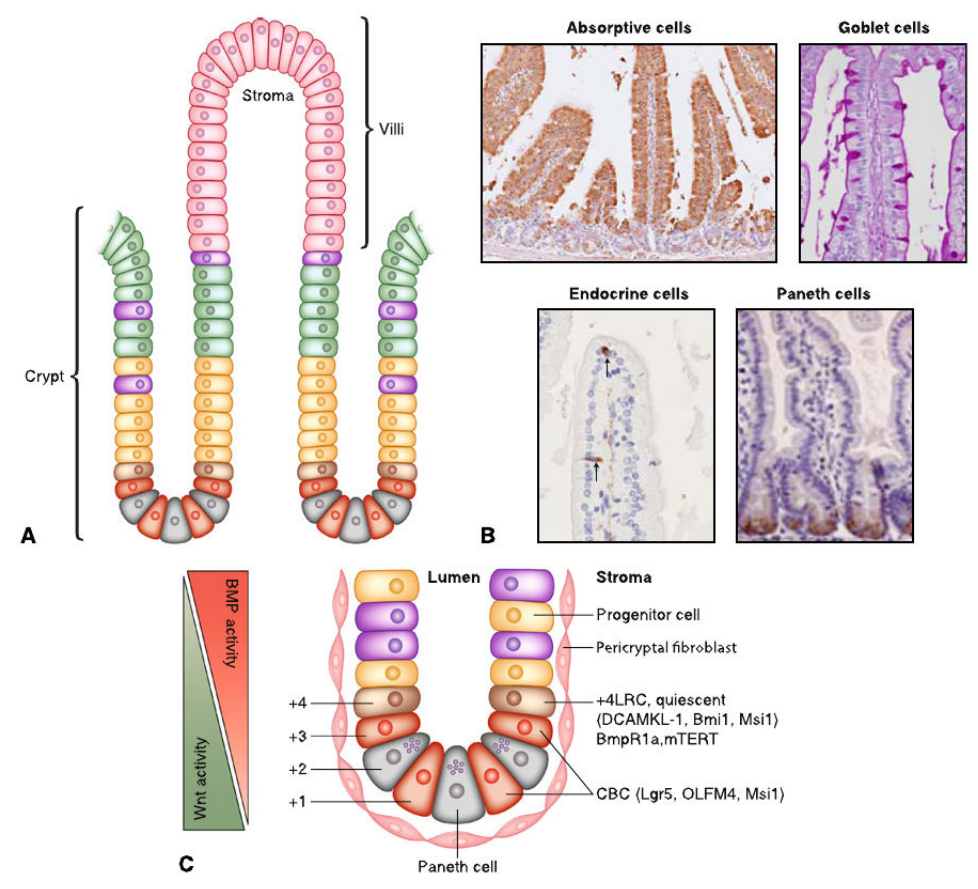

Fig. 1.

Intestinal stem cell (ISC) compartments in the mammalian intestine. a The intestinal cryptvillus unit. ISCs reside at the base of the crypt, either in the +4 position counting from the bottom of the crypt (brown) directly above the Paneth cells (gray), or as crypt base columnar (CBC) cells ( red) located between the Paneth cells, whereas transit-amplifying (TA) progenitor cells (yellow) can arise from self-renewing CBCs. Goblet cells and enterocytes are labeled purple and green, respectively. b Chemical and immunohistochemical detection of the four principal cell lineages of the small intestine: villus-associated absorptive cells (Fatty acid binding protein, stains the villi), Goblet cells (stained by periodic acid/Schiff), enteroendocrine cells (stained for synaptophysin, arrows), and Paneth cells (stained for lysozyme). c An enlarged view of a small intestinal crypt depicting two different stem cell regions; a quiescent stem cell zone ( +4 position), and an active stem cell zone ( +1 to +4 positions) scattered between the Paneth cells. The +4 label-retaining cells (LRCs) are normally maintained in a quiescent state through direct interaction with and signals generated from the niche, such as pericryptal fibroblast/myofibroblasts and adjacent enteroendocrine cells within the +4 region. CBCs, continuously activated by signals generated from stromal cells at the crypt base, are responsible for most of the regenerative capacity of the intestine under homeostatic conditions. A gradient of BMP signaling, known to inhibit proliferation, is established along the crypt-villus axis, with relatively high activity throughout the villus and correspondingly less activity within the crypt. An opposite gradient of Wnt signaling, providing an important proliferative stimulus, is highest at the crypt base and decreases toward the crypt-villus junction. (A, reproduced with permission from Dr. Hans Clevers, Hubrecht Institute-Royal Netherlands Academy of Arts and Sciences and the University Medical Center Utrecht, Utrecht, The Netherlands) 
Table 1

\section{Proposed intestinal stem cell markers}

\begin{tabular}{lll}
\hline Study & Marker & Function \\
\hline Kayahara et al. [39], Potten et al. [40] & Musashi-1 & RNA binding protein \\
Barker et al. [51••] & Lgr-5 & Unknown; Wnt target gene \\
Sangiorgi and Capecchi [55••] & Bmi-1 & Maintenance of somatic stem cells \\
Zhu et al. [53] & Prominin-1 & Self-renewal; tumor angiogenesis \\
May et al. [56••] & DCAMKL-1 & Label-retaining +4 cell; kinase \\
Batlle et al. [37] & Ephrin B3 & Stem/progenitor cell proliferation, migration, and polarity \\
He et al. [20] & p-PTEN & Colocalizes with label-retaining +4 cells \\
Tian et al. [41] & p-AKT & Label-retaining +4 cell; intestinal homeostasis \\
He et al. [20] & BMPR-1 $\alpha$ & Tumor suppressor/regulator of PTEN \\
Breault et al. [47] & mTERT & Label-retaining +4 cell \\
\hline
\end{tabular}

Curr Gastroenterol Rep. Author manuscript; available in PMC 2011 October 1. 